Igor V. Dolgachev · JongHae Keum

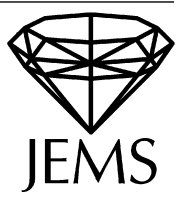

\title{
K3 surfaces with a symplectic automorphism of order 11
}

Received August 31, 2006 and in revised form September 22, 2007

\begin{abstract}
We classify possible finite groups of symplectic automorphisms of K3 surfaces of order divisible by 11 . The characteristic of the ground field must be equal to 11 . The complete list of such groups consists of five groups: the cyclic group $C_{11}$ of order $11, C_{11} \rtimes C_{5}, \mathrm{PSL}_{2}\left(\mathbb{F}_{11}\right)$ and the Mathieu groups $M_{11}, M_{22}$. We also show that a surface $X$ admitting an automorphism $g$ of order 11 admits a $g$-invariant elliptic fibration with the Jacobian fibration isomorphic to one of explicitly given elliptic K3 surfaces.
\end{abstract}

\section{Introduction}

Let $X$ be a K3 surface over an algebraically closed field $k$ of characteristic $p \geq 0$. An automorphism $g$ of $X$ is called symplectic if it preserves a regular 2-form of $X$. In positive characteristic $p$, an automorphism of order a power of $p$ is called wild. A wild automorphism is symplectic. A subgroup $G$ of the automorphism group $\operatorname{Aut}(X)$ is called symplectic if all elements of $G$ are symplectic, and wild if it contains a wild automorphism. No K3 surface in characteristic $p>11$ can admit a wild automorphism ([4, Theorem 2.1]). The existence of such a bound is not a common property among all classes of surfaces, e.g. in every positive characteristic one can find a rational surface and an abelian surface admitting a wild automorphism.

It is a well-known result of V. Nikulin that the order of a symplectic automorphism of finite order of a complex $\mathrm{K} 3$ surface takes value in the set $\{1,2,3,4,5,6,7,8\}$. This result is true over an algebraically closed field $k$ of positive characteristic $p$ if the order is coprime to $p$. The latter condition is automatically satisfied if $p>11$ [4]. If $p=11$, an elliptic K3 surface $X_{\varepsilon}$ defined by the equation of degree 12 in $\mathbb{P}(1,1,4,6)$

$$
y^{2}+x^{3}+\varepsilon x^{2} t_{0}^{4}+t_{0} t_{1}^{11}-t_{0}^{11} t_{1}=0, \quad \varepsilon \in k,
$$

admits a symplectic automorphism of order 11

$$
g_{\varepsilon}:\left(t_{0}, t_{1}, x, y\right) \mapsto\left(t_{0}, t_{0}+t_{1}, x, y\right) .
$$

I. V. Dolgachev: Department of Mathematics, University of Michigan, Ann Arbor, MI 48109, USA; e-mail: idolga@umich.edu

J. Keum: School of Mathematics, Korea Institute for Advanced Study, Seoul 130-722, Korea; e-mail: jhkeum@kias.re.kr

Mathematics Subject Classification (2000): 14J28, 14J50, 20D08 
The main result of the paper is the following.

Theorem 1.1. Let $G$ be a finite group of symplectic automorphisms of a K3 surface $X$ over an algebraically closed field of characteristic $p \geq 0$. Assume that the order of $G$ is divisible by 11. Then $p=11$ and $G$ is isomorphic to one of the following five groups:

$$
C_{11}, \quad C_{11} \rtimes C_{5}, \quad \operatorname{PSL}_{2}\left(\mathbb{F}_{11}\right), \quad M_{11}, \quad M_{22},
$$

where $C_{n}$ is the cyclic group of order $n$ and $M_{n}$ is one of the Mathieu groups. Moreover, the following assertions are true.

(i) For any element $g \in G$ of order $11, X$ admits a $g$-invariant elliptic pencil $|F|$ and $X$ is $C_{11}$-equivariantly isomorphic to a torsor of one of the surfaces $X_{\varepsilon}$ equipped with its standard elliptic fibration.

(ii) If $X=X_{\varepsilon}$ and $G$ contains an element of order 11 leaving invariant both the standard elliptic fibration and a section, then $G \cong C_{11}$ if $\varepsilon \neq 0$, and $G$ is isomorphic to a subgroup of $\mathrm{PSL}_{2}\left(\mathbb{F}_{11}\right)$ if $\varepsilon=0$.

The surface $X_{0}$ is a supersingular K3 surface with Artin invariant 1 isomorphic to the Fermat surface

$$
x_{0}^{4}+x_{1}^{4}+x_{2}^{4}+x_{3}^{4}=0 .
$$

In a recent paper of Kondō [7] it is proven that both $M_{11}$ and $M_{22}$ appear as symplectic automorphism groups of $X_{0}$. Thus the surface $X_{0}$ admits three maximal finite simple symplectic groups of automorphisms, isomorphic to $\mathrm{PSL}_{2}\left(\mathbb{F}_{11}\right), M_{11}$ and $M_{22}$. An element $g$ of order $p=11$ in the latter two groups leaves invariant an elliptic pencil. We do not know whether the pencil has a section; all we know is that it does not have a $g$-invariant section.

In our earlier work [4] finite groups of symplectic automorphisms of K3 surfaces in positive characteristic $p$ have been studied. If such a group is not wild, then it is isomorphic to a subgroup of the Mathieu group $M_{23}$ with at least four orbits in its natural action on a set of 24 elements. In characteristic $p<7$, there are examples of finite wild symplectic groups which cannot be embedded into $M_{23}$. In characteristic $p=11$, Theorem 1.1 shows that every finite symplectic group, wild or not, can be embedded into $M_{23}$. We do not know whether the same holds true in characteristic $p=7$.

Corollary 1.2. A finite group $G$ acts symplectically and wildly on a K3 surface over an algebraically closed field of characteristic 11 if and only if $G$ is isomorphic to a subgroup of $M_{23}$ of order divisible by 11 and having three or four orbits in its natural action on $a$ set of 24 elements.

\section{Notation}

For an automorphism group $G$ or an automorphism $g$ of $X$, we denote by $X^{g}$ the fixed locus with reduced structure, i.e. the set of fixed points of $g$.

A subset $T$ of $X$ is $G$-invariant if $g(T)=T$ for all $g \in G$. In this case we say $G$ leaves $T$ invariant. 
An elliptic pencil $|F|$ on $X$ is $G$-invariant if $g(F) \in|F|$ for all $g \in G$. In this case we say $G$ leaves $|F|$ invariant.

We also use the following notations for groups:

- $C_{n}$ the cyclic group of order $n$, sometimes denoted by $n$,

- $m: n=m \rtimes n$ the semidirect product of cyclic groups $C_{m}$ and $C_{n}$,

- $M_{n}$ the Mathieu group of degree $n$,

- $L_{n}(q)=\operatorname{PSL}_{n}\left(\mathbb{F}_{q}\right)$,

- $\mathrm{GU}_{n}(q)$ the general unitary group over $\mathbb{F}_{q}$,

- \# $G$ the cardinality of a group $G$,

- $V^{g}$ the subspace of $g$-invariant vectors of a vector space $V$.

\section{Wild $C_{11}$-actions on $\mathrm{K3}$ surfaces}

In this section we improve the result of [3] and [4] in the case of wild $C_{11}$-actions on $\mathrm{K} 3$ surfaces.

Lemma 2.1. Let $X$ be a $K 3$ surface over an algebraically closed field $k$ of characteristic 11. Assume that $X$ admits an automorphism $g$ of order 11 . Then X admits a g-invariant elliptic pencil $|F|$.

Proof. By Theorem 2.2 and Propositions 2.3, 2.5 of [4], we see that the fixed locus $X^{g}$ is either the support of a fibre of an elliptic fibration, or a point. Thus we need to prove the lemma in the second case.

Assume that $X^{g}$ is a point. In this case, by Theorems 1 and 2.4 of [3] (or Proposition 2.4 of [4]), the quotient surface $X /\langle g\rangle$ is a rational surface with trivial canonical divisor and one isolated elliptic Gorenstein singularity. By Proposition 2.9 of [4], $X$ admits a $g$-invariant elliptic pencil.

Lemma 2.2. Let $X$ be a $K 3$ surface over an algebraically closed field $k$ of characteristic 11. Assume that $X$ admits an automorphism $g$ of order 11 . Let $\phi: X \rightarrow \mathbb{P}^{1}$ be $a$ $g$-invariant elliptic fibration. Then the following assertions are true.

(i) $\phi$ has either 12 cuspidal fibres, or one cuspidal fibre and 22 nodal fibres.

(ii) $g^{*}$ acts on the base $\mathbb{P}^{1}$ faithfully, leaving one cuspidal fibre $F_{0}$ invariant and has either one orbit or two orbits on the set of remaining singular fibres.

(iii) $X^{g}$ is either the whole curve $F_{0}$ or the cusp of $F_{0}$.

Proof. Assume that $g^{*}$ acts as identity on the base $\mathbb{P}^{1}$. Then $g$ becomes an automorphism of the elliptic curve, $X / \mathbb{P}^{1}$ over the function field of $\mathbb{P}^{1}$. On the Jacobian $J$ of this elliptic curve, $g$ induces an automorphism $g^{\prime}$ of order 11 . Note that $J$ is an elliptic K3 surface with a section (cf. [2, Theorem 5.7.2]). Since the order of $g^{\prime}$ is greater than $3, g^{\prime}$ must be a translation by an 11-torsion section. This contradicts Theorem 2.13 of [4] stating that no jacobian elliptic K3 surface in characteristic $p$ admits a nontrivial $p$-torsion if $p>7$. Thus $g^{*}$ acts on the base $\mathbb{P}^{1}$ faithfully, and hence fixes only one point. Let $F_{0}$ be the 
corresponding fibre. The fibre $F_{0}$ contains $X^{g}$. An elliptic K3 surface cannot have only one singular fibre. This follows from the equality $e(X)=24$ for the Euler number and the bound $\rho(X) \leq 22$ for the Picard number. Singular fibres away from $F_{0}$ form orbits of fibres of the same type, and the number of such singular fibres is a multiple of 11 , the order of $g$. Thus the Euler number of such a fibre must be $\leq 2$. This leaves the following four possibilities:

(a) one orbit of singular fibres of type $I_{2}$,

(b) one orbit of singular fibres of type $I I$,

(c) one orbit of singular fibres of type $I_{1}$,

(d) two orbits of singular fibres of type $I_{1}$.

Let $Y$ be a minimal resolution of $X /\langle g\rangle$ and $Z$ the relative minimal model of $Y$. Then $Z$ is a rational elliptic surface with $e(Z)=12$ and $\rho(Z)=10$. The rationality follows from [3. Theorems 1 and 3.7].

In case (a), $Z$ has two singular fibres: one fibre of type $I_{2}$ and the other fibre $Z_{0}$ coming from $F_{0}$. The fibre $Z_{0}$ has Euler number $e\left(Z_{0}\right)=10$ and eight irreducible components. Such an elliptic fibre does not exist. Thus case (a) does not occur.

In case (b), $Z$ has two singular fibres: one fibre of type $I I$ and the other fibre $Z_{0}$ coming from $F_{0}$. The fibre $Z_{0}$ has Euler number $e\left(Z_{0}\right)=10$ and nine irreducible components, hence is of type $\tilde{D}_{8}$ or $\tilde{E}_{8}$, additive in both cases. It follows that $F_{0}$ cannot be of multiplicative type $I_{2}$, hence it is of type $I I$.

In case (c), $Z$ has two singular fibres; one fibre of type $I_{1}$ and the other fibre $Z_{0}$ coming from $F_{0}$. The fibre $Z_{0}$ has Euler number $e\left(Z_{0}\right)=11$ and nine irreducible components. Such an elliptic fibre does not exist. Thus case (c) does not occur.

In case (d), $Z$ has three singular fibres; two fibres of type $I_{1}$ and the fibre $Z_{0}$ coming from $F_{0}$. The fibre $Z_{0}$ has Euler number $e\left(Z_{0}\right)=10$ and has nine irreducible components, hence is of type $\tilde{D}_{8}$ or $\tilde{E}_{8}$, additive in both cases. By the same reason as in case (b), $F_{0}$ is of type $I I$. This completes the proof of (i) and (ii). The action of $g$ on the rational curve $F_{0}$ gives (iii).

Lemma 2.3. Let $X$ be a $K 3$ surface over an algebraically closed field $k$ of characteristic 11. Assume that $X$ admits an automorphism $g$ of order 11. Then the following assertions are true.

(i) $\operatorname{rank} \operatorname{Pic}(X /\langle g\rangle)=2$.

(ii) For any $l \neq 11, \operatorname{dim} H_{\mathrm{et}}^{2}\left(X, \mathbb{Q}_{l}\right)^{g}=\operatorname{rank} \operatorname{Pic}(X)^{g}=2$.

(iii) $\operatorname{rank} \operatorname{Pic}(X)=2,12$ or 22 .

Proof. By Lemma 2.1, $X$ admits a $g$-invariant elliptic fibration. The quotient surface $X /\langle g\rangle$ is rational (Theorems 1 and 3.7 of [3]), and has no reducible fibre (Lemma 2.2). Let $Y$ be a minimal resolution of $X /\langle g\rangle$. Then $Y$ is an extremal rational elliptic surface with only one reducible fibre, not necessarily relatively minimal. Thus the sublattice $N$ of $\operatorname{Pic}(Y)$ spanned by the exceptional divisor classes has corank 2 . This proves (i).

Recall that for a nonsingular projective variety $Z$ in positive characteristic, there is an exact sequence of $\mathbb{Q}_{l}$-vector spaces

$$
0 \rightarrow \mathrm{NS}(Z) \otimes \mathbb{Q}_{l} \rightarrow H_{\mathrm{et}}^{2}\left(Z, \mathbb{Q}_{l}\right) \rightarrow T_{l}^{2}(Z) \rightarrow 0,
$$


where $T_{l}^{2}(Z)=T_{l}(\operatorname{Br}(Z))$ in the standard notation in the theory of étale cohomology. The Brauer group $\operatorname{Br}(X)$ is known to be a birational invariant, in particular, it is trivial when $Z$ is a rational variety. In fact, one can show that

$$
\begin{aligned}
\mathrm{NS}(Z) \otimes \mathbb{Q}_{l} & =\operatorname{Ker}\left(H_{\mathrm{et}}^{2}\left(Z, \mathbb{Q}_{l}\right) \rightarrow H^{2}\left(k(Z), \mathbb{Q}_{l}\right)\right), \\
T_{l}^{2}(Z) & =\operatorname{Im}\left(H_{\mathrm{et}}^{2}\left(Z, \mathbb{Q}_{l}\right) \rightarrow H^{2}\left(k(Z), \mathbb{Q}_{l}\right)\right) .
\end{aligned}
$$

Here $H^{2}\left(k(Z), \mathbb{Q}_{l}\right)=\lim _{U} H^{2}\left(U, \mathbb{Q}_{l}\right)$, where $U$ runs through the set of open subsets of $Z$ (see [15]). It is known that the dimensions of all $\mathbb{Q}_{l}$-spaces above do not depend on $l$ prime to the characteristic. Since $Y$ is rational, the Lefschetz number $\lambda(Y)$ equals $\operatorname{dim} T_{l}^{2}(Y)=0$. It follows from [15, Proposition 5] that

$$
T_{l}^{2}(X)^{g} \cong T_{l}^{2}(Y)=0 .
$$

Hence,

$$
\operatorname{dim} H_{\mathrm{et}}^{2}\left(X, \mathbb{Q}_{l}\right)^{g}=\operatorname{rank} \mathrm{NS}(X)^{g}=\operatorname{rank} \operatorname{Pic}(X)^{g}=\operatorname{rank} \operatorname{Pic}(X /\langle g\rangle)=2 .
$$

This proves (ii).

Considering the $\mathbb{Q}$-representation of the cyclic group $\langle g\rangle$ of order 11 on $\operatorname{Pic}(X) \otimes \mathbb{Q}$, we get (iii) from (ii).

\section{The surfaces $X_{\varepsilon}$}

Let $p=11$ and $X_{\varepsilon}$ be the $\mathrm{K} 3$ surface from 1.1 . The surface $X_{\varepsilon}$ has an elliptic pencil defined by the projection to the $t_{0}, t_{1}$ coordinates

$$
f_{\varepsilon}: X_{\varepsilon} \rightarrow \mathbb{P}^{1} .
$$

We will refer to it as the standard elliptic fibration. Its zero section, the section at infinity, will be denoted by $S_{\varepsilon}$. It is immediately checked that the surface $X_{\varepsilon}$ is nonsingular. Computing the discriminant $\Delta_{\varepsilon}$ of the Weierstrass equation of the general fibre of the elliptic fibration on $X_{\varepsilon}$ we find that

$$
\Delta_{\varepsilon}=-t_{0}^{2}\left(t_{1}^{11}-t_{0}^{10} t_{1}\right)\left(5 t_{1}^{11}-5 t_{0}^{10} t_{1}+4 \varepsilon^{3} t_{0}^{11}\right) .
$$

This shows that the set of singular fibres of the elliptic fibration on $X_{0}$ (resp. $X_{\varepsilon}, \varepsilon \neq 0$ ) consists of 12 irreducible cuspidal curves (resp. one cuspidal fibre and 22 nodal fibres). The automorphism $g_{\varepsilon}$ given by (1.2) is symplectic and of order 11. It fixes pointwise the cuspidal fibres over the point $\infty=(0,1)$ and has one orbit (resp. two orbits) on the set of remaining singular fibres. It leaves invariant the zero section $S_{\varepsilon}$. The quotient surface $X_{\varepsilon} /\left\langle g_{\varepsilon}\right\rangle$ is a rational elliptic surface with a double rational point of type $E_{8}$ equal to the image of the singular point of the fixed fibre. A minimal resolution of the surface has one reducible nonmultiple fibre of type $\tilde{E}_{8}$ and one irreducible singular cuspidal fibre (resp. two nodal fibres). 
Proposition 3.1. Let $X$ be a $K 3$ surface over an algebraically closed field $k$ of characteristic 11. Assume that $X$ admits an automorphism $g$ of order 11 . Let $f: X \rightarrow \mathbb{P}^{1}$ be a g-invariant elliptic fibration. Assume that $f$ has a section $S$. Then there exists an isomorphism $\phi: X \rightarrow X_{\varepsilon}$ of elliptic surfaces such that $\phi g \phi^{-1}=\tau g_{\varepsilon}$ for some translation automorphism $\tau$ of $X_{\varepsilon}$. In particular, if $g(S)=S$ then $\phi g \phi^{-1}=g_{\varepsilon}$.

Proof. Let

$$
y^{2}+x^{3}+A\left(t_{0}, t_{1}\right) x+B\left(t_{0}, t_{1}\right)=0
$$

be the Weierstrass equation of the $g$-invariant elliptic pencil, where $A$ (resp. $B$ ) is a binary form of degree 8 (resp. 12). By Lemma 2.2. $g$ acts nontrivially on the base of the fibration. After a linear change of the coordinates $\left(t_{0}, t_{1}\right)$ we may assume that $g$ acts on the base by

$$
g:\left(t_{0}, t_{1}\right) \mapsto\left(t_{0}, t_{1}+t_{0}\right) .
$$

We know that a $g$-invariant elliptic fibration has one $g$-invariant irreducible cuspidal fibre $F_{0}$ and either 22 irreducible nodal fibres forming two orbits, or 11 irreducible cuspidal fibres forming one orbit (Lemma 2.2). Thus the discriminant polynomial $\Delta=-4 A^{3}-$ $27 B^{2}$ must have one double root (corresponding to the fibre $F_{0}$ ) and either one orbit of double roots, or two orbits of simple roots. We know that the zeros of $A$ correspond to either cuspidal fibres or nonsingular fibres with "complex multiplication" automorphism of order 6. Since this set is invariant with respect to our automorphism of order 11 acting on the base, we see that the only possibility is $A=c t_{0}^{8}$ for some constant $c \in k$. We obtain $\Delta=-4 c^{3} t_{0}^{24}-27 B^{2}$. Again this uniquely determines $B$ and hence the surface. Since $B$ is of degree 12 and invariant under the action of $g$ on the base, it must be of the form

$$
B=a\left(t_{1}^{11}-t_{0}^{10} t_{1}\right) t_{0}+b t_{0}^{12}
$$

for some constants $a, b$. One can rewrite the above Weierstrass equation in the form

$$
y^{2}+x^{3}+\varepsilon x^{2} t_{0}^{4}+a\left(t_{0} t_{1}^{11}-t_{0}^{11} t_{1}\right)+b^{\prime} t_{0}^{12}=0 .
$$

A suitable linear change of variables $u_{0}=t_{0}, u_{1}=t_{1}+d t_{0}$ makes $b^{\prime}=0$ without changing the action of $g$ on the base. Thus $X \cong X_{\varepsilon}$ as an elliptic surface. Let $\phi: X \rightarrow X_{\varepsilon}$ be the isomorphism. The composite

$$
\phi g \phi^{-1} g_{\varepsilon}^{-1}: X_{\varepsilon} \rightarrow X_{\varepsilon}
$$

acts trivially on the base, hence must be a translation automorphism. Since $\phi$ maps the zero section $S$ of $f: X \rightarrow \mathbb{P}^{1}$ to the zero section $S_{\varepsilon}$ of $f_{\varepsilon}: X_{\varepsilon} \rightarrow \mathbb{P}^{1}$ and $g_{\varepsilon}\left(S_{\varepsilon}\right)=S_{\varepsilon}$, the last assertion follows.

Lemma 3.2. Let $\varepsilon=0$. For any translation automorphism $\tau$ of $X_{0}$, the composite automorphisms $\tau g_{0}$ and $g_{0} \tau$ are of order 11 . 
Proof. Let $f: X \rightarrow B$ be any elliptic surface with a section $S$. Recall that its MordellWeil group MW $(f)$ is isomorphic to the quotient of the Néron-Severi group by the subgroup generated by the divisor classes of $S$ and the components of fibres. Thus any automorphism $g$ of $X$ which preserves the class of a fibre and the section $S$ acts linearly on the group $\mathrm{MW}(f)$. Assume $\mathrm{MW}(f)$ is torsion free. Suppose $g$ is of finite order $n$ with rank MW $(f)^{g}=0$ and let $\tau$ be a translation automorphism identified with an element of $\operatorname{MW}(f)$. Then for any $s \in \mathrm{MW}(f)$ we have

$$
\tau g(s)=g(s)+\tau, \quad(\tau g)^{n}(s)=g^{n}(s)+g^{n-1}(\tau)+\cdots+g(\tau)+\tau=s .
$$

The last equality follows from the fact that the linear action of $g-1_{X}$ on $\operatorname{MW}(f)$ is invertible. This shows that $(\tau g)^{n}$ acts identically on $\mathrm{MW}(f)$. It also acts identically on the class of a fibre. Thus $(\tau g)^{n}$ acts identically on the Néron-Severi lattice.

Apply this to our case $\varepsilon=0$, when $g=g_{0}$ is a symplectic automorphism of order 11 of $X_{0}$. We will see in the proof of Proposition 3.8 that MW $\left(f_{0}\right)$ is torsion free. By Lemma 2.3 (ii), rank $\mathrm{MW}\left(f_{0}\right)^{g}=0$. Since the surface $X_{0}$ is supersingular (see Remark 3.6, by a theorem of Ogus [10], an automorphism acting identically on the Picard group must be the identity. Thus $\tau g_{0}$ is a symplectic automorphism of order 11 for any section $\tau$.

An interesting question: Is there a $\tau$ such that the fixed locus $X_{0}^{\tau g_{0}}$ consists of an isolated point, the cusp of a cuspidal curve fixed pointwise by $g_{0}$ ? We do not know any example of a symplectic automorphism of order 11 with an isolated fixed point.

Corollary 3.3. Let X be a K3 surface over an algebraically closed field $k$ of characteristic 11. Assume that $X$ admits an automorphism $g$ of order 11. Then $X$ is isomorphic to a torsor of one of the elliptic surfaces $X_{\varepsilon}$. The order of this torsor in the Shafarevich-Tate group of torsors is equal to 1 or 11.

Proof. Let $f_{J}: J \rightarrow \mathbb{P}^{1}$ be the Jacobian fibration of the elliptic fibration $f: X \rightarrow \mathbb{P}^{1}$ defined by the $g$-invariant elliptic pencil. Let $J^{o}$ be the open subset of $J$ whose complement is the set of singular fibres of $f_{J}$. We know that the fibres of $f$ are irreducible. By a result of M. Raynaud, this allows us to identify $J^{o}$ with the component $\mathbf{P i c}_{X / \mathbb{P}^{1}}^{0}$ of the relative Picard scheme of invertible sheaves of degree 0 (see [2, Proposition 5.2.2]). The automorphism $g$ acts naturally on the Picard functor and hence on $J^{o}$. Since $J$ is minimal, it acts biregularly on $J$. This action preserves the elliptic fibration on $J$ and defines an automorphism of order 11 on the base. This implies that there exists a $C_{11}$-equivariant isomorphism of elliptic surfaces $J$ and $X_{\varepsilon}$.

The assertion about the order of the torsor follows from the existence of a section or an 11-section of $f$. In fact, let $Y$ be a nonsingular relatively minimal model of the elliptic surface $X /\langle g\rangle$ with the elliptic fibration induced by $f$. It is a rational elliptic surface. Let $F_{0}$ be the $g$-invariant fibre of $f$ over a point $s_{0} \in \mathbb{P}^{1}$. The singular fibres of the elliptic fibration $f^{\prime}: Y \rightarrow \mathbb{P}^{1}$ over $\mathbb{P}^{1} \backslash\left\{s_{0}\right\}$ are either two irreducible nodal fibres $(\varepsilon \neq 0)$ or one cuspidal irreducible fibre $(\varepsilon=0)$. The standard argument in the theory of elliptic surfaces shows that the fibre of $f^{\prime}$ over $s_{0}$ is either of type $\tilde{E}_{8}$ or $\tilde{D}_{8}$. This fibre is not multiple if and only if $f^{\prime}$ has a section. The pre-image of this section is a section of $f$ making $X$ the 
trivial torsor. A singular fibre of additive type can be multiple only if the characteristic is positive, and the multiplicity $m$ must be equal to the characteristic (see [2, Proposition 5.1.5]). In this case an exceptional curve of the first kind on $Y$ is an $m$-section. The preimage of this multi-section on $X$ is an $m$-section, in our case an 11-section.

Remark 3.4. Note that, even in the case $X=X_{\varepsilon}$, the $g$-invariant fibration may be different from the standard elliptic fibration. In other words, a nontrivial torsor of an elliptic surface could be isomorphic to the same surface. This strange phenomenon could happen only in positive characteristic and only for torsors of order divisible by the characteristic. We do not know an example where this strange phenomenon really occurs. In Kondō's example, the $g$-invariant elliptic fibration for an element $g$ of order 11 in $G=M_{11}$ or $M_{22}$ may have a section (but no $g$-invariant section!). If this happens, it is isomorphic to the standard elliptic fibration and hence $g$ is conjugate to $\tau g_{\varepsilon}$, as we have seen in Proposition 3.1 .

Lemma 3.5. Suppose $p=11$. Then there is a finite subgroup $K_{\varepsilon}$ of $\operatorname{Aut}\left(X_{\varepsilon}\right)$ with the following property:

(i) $K_{\varepsilon}$ leaves invariant both the standard elliptic fibration of $X_{\varepsilon}$ and the zero section $S_{\varepsilon}$ which is the section at infinity.

(ii) $K_{0} \cong \mathrm{GU}_{2}(11) /( \pm I) \cong L_{2}(11): 12$ and $K_{1} \cong 11: 4$, where the first factor in the semidirect product is a symplectic subgroup and the second factor a nonsymplectic subgroup.

(iii) The image of $K_{\varepsilon}$ in $\operatorname{Aut}\left(\mathbb{P}^{1}\right)$ is equal to the subgroup $\operatorname{Aut}\left(\mathbb{P}^{1}, V\left(\Delta_{\varepsilon}\right)\right)$ which leaves the set $V\left(\Delta_{\varepsilon}\right)$ invariant.

(iv) $\operatorname{Aut}\left(\mathbb{P}^{1}, V\left(\Delta_{0}\right)\right) \cong \operatorname{PGU}_{2}(11) \cong L_{2}(11) .2$ and $\operatorname{Aut}\left(\mathbb{P}^{1}, V\left(\Delta_{\varepsilon}\right)\right) \cong 11: 2$ if $\varepsilon \neq 0$.

Proof. Assume $\varepsilon=0$. After a linear change of variables

$$
t_{0}=\alpha^{11} t_{0}^{\prime}+\alpha t_{1}^{\prime}, \quad t_{1}=t_{0}^{\prime}+t_{1}^{\prime},
$$

where $\alpha \in \mathbb{F}_{11^{2}} \backslash \mathbb{F}_{11} \subset k^{*}$, we can transform the polynomial $t_{0} t_{1}^{11}-t_{0}^{11} t_{1}$ to the form $\lambda t_{0}^{12}+\mu t_{1}^{12}$. After scaling, we may assume that $f=t_{0}^{12}+t_{1}^{12}$. Now notice that $f$ represents a hermitian form over the field $\mathbb{F}_{11^{2}}$, hence the finite unitary group $\mathrm{GU}_{2}(11)$ leaves the polynomial $f$ invariant. The group $\mathrm{GU}_{2}(11)$ acts on the surface

$$
X_{0} \cong V\left(y^{2}+x^{3}+t_{0}^{12}+t_{1}^{12}\right)
$$

in an obvious way, by acting on the variables $t_{0}, t_{1}$ and identically on the variables $x, y$. Note that

$$
\left(t_{0}, t_{1}, x, y\right)=\left(\lambda t_{0}, \lambda t_{1}, \lambda^{4} x, \lambda^{6} y\right)
$$

in $\mathbb{P}(1,1,4,6)$ for all $\lambda \in k^{*}$. In particular, $\left(t_{0}, t_{1}, x, y\right)=\left(-t_{0},-t_{1}, x, y\right)$, so $-I \in$ $\mathrm{GU}_{2}(11)$ acts trivially on $X_{0}$. Note also that $\mathrm{SU}_{2}(11)$ and hence $\mathrm{PSU}_{2}(11)$ acts symplectically on $X_{0}$. The action of $\mathrm{PSU}_{2}(11)$ is faithful because it is a simple group. Take $K_{0}=\mathrm{GU}_{2}(11) /( \pm I)$ and consider the homomorphism

$$
\operatorname{det}: K_{0} \rightarrow\left(\mathbb{F}_{11^{2}}\right)^{*} \text {. }
$$


It is known that

$$
\mathrm{PSU}_{2}(11) \cong \mathrm{PSL}_{2}\left(\mathbb{F}_{11}\right)=L_{2}(11) .
$$

If $A \in \mathrm{GU}_{2}(11)$, then $(\operatorname{det} A)^{12}=(\operatorname{det} A)(\overline{\operatorname{det} A})=\operatorname{det} A^{t} \bar{A}=\operatorname{det} I=1$, so the image of det is a cyclic group of order dividing 12. On the other hand, if $\zeta \in \mathbb{F}_{11^{2}}$ is a 12-th root of unity, the unitary matrix $\left(\begin{array}{ll}1 & 0 \\ 0 & \zeta\end{array}\right)$ generates an order 12 subgroup of $K_{0}$, which acts on $X_{0}$ nonsymplectically. This proves (i) and (ii).

We know that the group $\mathrm{GU}_{2}(11)$ leaves the polynomial $f$ invariant. Thus its image $\mathrm{PGU}_{2}(11)$ in $\operatorname{Aut}\left(\mathbb{P}^{1}\right)$ must coincide with $\operatorname{Aut}\left(\mathbb{P}^{1}, V\left(\Delta_{0}\right)\right)$. It is known that $\mathrm{PGU}_{2}(11)$ is a maximal subgroup in the permutation group $\mathfrak{S}_{12}$ and $\operatorname{PGU}_{2}(11) \cong \operatorname{PGL}_{2}\left(\mathbb{F}_{11}\right) \cong$ $L_{2}(11) .2$, a nonsplit extension. The quotient group is generated by the image of the automorphism $\left(t_{0}, t_{1}\right) \mapsto\left(t_{0}, \zeta t_{1}\right)$, where $\zeta \in \mathbb{F}_{11^{2}}$ is a 12-th root of unity. This proves (iii) and (iv).

Assume $\varepsilon \neq 0$. An element of $\mathrm{PGL}_{2}(k)$ leaving $V\left(\Delta_{\varepsilon}\right)$ invariant must either leave all factors of $\Delta_{\varepsilon}$ from (3.1) invariant or interchange the second and the third factors. It can be seen by computation that the group $\operatorname{Aut}\left(\mathbb{P}^{1}, V\left(\Delta_{\varepsilon}\right)\right)$ is generated by the following two automorphisms:

$$
e\left(t_{0}, t_{1}\right)=\left(t_{0}, t_{1}+t_{0}\right), \quad i\left(t_{0}, t_{1}\right)=\left(t_{0},-t_{1}+b t_{0}\right)
$$

where $b$ is a root of $b^{11}-b+3 \varepsilon^{3}=0$. The order of $e$ (resp. $i$ ) is 11 (resp. 2) and $i$ normalizes $e$. We see that they lift to automorphisms of $X_{\varepsilon}$

$$
\begin{aligned}
& \tilde{e}\left(t_{0}, t_{1}, x, y\right)=\left(t_{0}, t_{1}+t_{0}, x, y\right), \\
& \tilde{i}\left(t_{0}, t_{1}, x, y\right)=\left(t_{0},-t_{1}+b t_{0},-x+3 \varepsilon t_{0}^{4}, \sqrt{-1} y\right),
\end{aligned}
$$

and we take $K_{\varepsilon}=(\tilde{e}, \tilde{i})$. Clearly $\tilde{i}$ is nonsymplectic of order 4 and normalizes $\tilde{e}$ which is symplectic of order 11, and both leave invariant the zero section $S_{\varepsilon}$.

Remark 3.6. The equation (3.2) makes $X_{0}$ a weighted Delsarte surface according to the definition in [5]. It follows that $X_{0}$ is a supersingular surface with Artin invariant $\sigma=1$ (loc.cit.). It is known that all such surfaces are isomorphic [10]. Thus $X_{0}$ is isomorphic to either the Fermat quartic

$$
x_{0}^{4}+x_{1}^{4}+x_{2}^{4}+x_{3}^{4}=0
$$

or the Kummer surface associated to the product of supersingular elliptic curves, or the modular elliptic surface of level 4 (see [14]). We do not know whether the surface $X_{\varepsilon}$, $\varepsilon \neq 0$, is supersingular. By Lemma 2.3, we know that $\operatorname{rank} \operatorname{Pic}\left(X_{\varepsilon}\right)=2,12$ or 22 .

Definition 3.7. The subgroup $K_{\varepsilon} \subset \operatorname{Aut}\left(X_{\varepsilon}\right)$ from Lemma 3.5 contains a symplectic subgroup leaving invariant the standard elliptic fibration of $X_{\varepsilon}$, isomorphic to $L_{2}(11)$ if $\varepsilon=0$ and to $C_{11}$ if $\varepsilon=1$. Denote this subgroup by $H_{\varepsilon}$. It leaves invariant the zero section $S_{\varepsilon}$ of the elliptic fibration. 
The group $H_{\varepsilon}$ acts on the base curve $\mathbb{P}^{1}$ and we have a homomorphism

$$
\pi: H_{\varepsilon} \rightarrow \operatorname{Aut}\left(\mathbb{P}^{1}, V\left(\Delta_{\varepsilon}\right)\right),
$$

which is an embedding. The image $\pi\left(H_{\varepsilon}\right)$ is equal to the unique index 2 subgroup of $\operatorname{Aut}\left(\mathbb{P}^{1}, V\left(\Delta_{\varepsilon}\right)\right)$.

Proposition 3.8. Let $G$ be a finite group of symplectic automorphisms of the surface $X_{\varepsilon}$ leaving invariant the standard elliptic fibration of $X_{\mathcal{\varepsilon}}$. Let

$$
\psi: G \rightarrow \operatorname{Aut}\left(\mathbb{P}^{1}, V\left(\Delta_{\varepsilon}\right)\right)
$$

be the natural homomorphism. Then $\psi$ is an embedding. If in addition $G$ is wild and leaves invariant the zero section $S_{\varepsilon}$, then $G$ is contained in $H_{\varepsilon}$.

Proof. Let $\alpha \in \operatorname{Ker}(\psi)$. Then $\alpha$ acts trivially on the base curve. Since $p>3$, $\alpha$ being symplectic must be a translation by a torsion section. It is known that there is no $p$-torsion in the Mordell-Weil group of an elliptic K3 surface if the characteristic $p$ is greater than 7 ([4, Theorem 2.13]), and there are no other torsion sections because no symplectic automorphism of order coprime to $p$ can have more than eight fixed points ([4], Theorem 3.3), while the fibration has 12 or 23 singular fibres. Hence $\alpha$ must be the identity automorphism. This proves that $\psi$ is an embedding.

If $\psi$ is surjective, then $\# G=2 \cdot \# L_{2}(11)$ or $2 \cdot 11$, which cannot be the order of a wild symplectic group in characteristic 11 , by Proposition 4.2 and Lemma 5.2. Thus $\psi$ is not surjective. From this we see that if $G$ is wild, then $\psi(G)$ is contained in the unique index 2 subgroup $\pi\left(H_{\varepsilon}\right)$ of $\operatorname{Aut}\left(\mathbb{P}^{1}, V\left(\Delta_{\varepsilon}\right)\right)$. If an element $\alpha \in G$ and an element $h \in H_{\varepsilon}$ have the same image in $\operatorname{Aut}\left(\mathbb{P}^{1}, V\left(\Delta_{\varepsilon}\right)\right)$, then $\alpha h^{-1}$ is a translation by a section. If $\alpha$ leaves invariant the zero section $S_{\varepsilon}$, so does $\alpha h^{-1}$, hence $\alpha h^{-1}$ is the identity automorphism. This proves the second assertion.

\section{A Mathieu representation}

From now on $X$ is a K3 surface over an algebraically closed field of characteristic $p=11$ and $G$ a group of symplectic automorphisms of $X$ of order divisible by 11 .

Lemma 4.1. Let $G$ be a finite group of symplectic automorphisms of a K3 surface X over an algebraically closed field of characteristic $p=11$. Then

$$
\operatorname{ord}(g) \in\{1,2,3,4,5,6,7,8,11\} \quad \text { for all } g \in G \text {. }
$$

Proof. If the order ord $(g)$ of $g \in G$ is coprime to the characteristic $p=11$, then $\operatorname{ord}(g) \in$ $\{1, \ldots, 8\}$ by Theorem 3.3 of [4]. It remains to show that $G$ cannot contain any element of order $11 r, r>1$. Assume the contrary, and let $h \in G$ be an element of order $11 r$. We may assume that $r$ is a prime and hence $r=2,3,5,7$, or 11. Let $g=h^{r}$. Then $g$ is of order 11. By Lemmas 2.1 and 2.3, $X$ admits a $g$-invariant elliptic pencil $|F|$ and $\operatorname{rank} \operatorname{Pic}(X)^{g}=2$. 
Case 1: $r=11$. In this case, let

$$
M:=\operatorname{Pic}(X)^{g} \cong \mathbb{Z}^{2} .
$$

Then $h$ acts on $M$. This action must be trivial, since a free $\mathbb{Z}$-module of rank 2 does not admit an automorphism of order 11. Thus $M=\operatorname{Pic}(X)^{h}$. Since the divisor class $[F]$ is contained in $M$, we see that the elliptic pencil $|F|$ is $h$-invariant. Note that by Lemma 2.2 the automorphism $g$ leaves only one fibre $F_{0}$ invariant. This implies that $h$ acts on the base curve $\mathbb{P}^{1}$ of the pencil $|F|$ faithfully. However, using the Jordan canonical form we see that $\mathbb{P}^{1}$ does not admit an automorphism of order $11^{2}$. A contradiction.

Case 2: $r=2,3,5,7$. Let $F_{0} \in|F|$ be the $g$-invariant fibre. Then $F_{0}$ is a cuspidal curve and $X^{g}$ is either the cusp of $F_{0}$ or the whole curve $F_{0}$ (Lemma 2.2). Let

$$
f=h^{11} \text {. }
$$

Then $f$ is of order $r$. By Theorem 3.3 of [4],

$$
3 \leq \# X^{f} \leq 8 .
$$

Since $r$ is prime to 11 ,

$$
X^{h}=X^{f} \cap X^{g} .
$$

Clearly $g$ acts on the finite set $X^{f}$, and this action cannot be of order 11 . This means that $g$ acts trivially on $X^{f}$, i.e. $X^{f} \subset X^{g}$. Thus $X^{g}$ cannot be a point and

$$
X^{h}=X^{f} \subset X^{g}=F_{0} .
$$

Now $h$ acts on $X^{g}=F_{0}$ with $\# X^{f}$ fixed points. But no nontrivial action on a rational curve can fix more than two points. A contradiction.

A Mathieu representation of a finite group $G$ is a 24-dimensional representation on a vector space $V$ over a field of characteristic zero with character

$$
\chi(g)=\epsilon(\operatorname{ord}(g))
$$

where

$$
\epsilon(n)=24\left(n \prod_{p \mid n}\left(1+\frac{1}{p}\right)\right)^{-1}, \quad \epsilon(1)=24 \text {. }
$$

The number

$$
\mu(G)=\frac{1}{\# G} \sum_{g \in G} \epsilon(\operatorname{ord}(g))
$$

is equal to the dimension of the subspace $V^{G}$ of $V$. Here $V^{G}$ is the linear subspace of vectors fixed by $G$. The natural action of a finite group $G$ of symplectic automorphisms of a complex K3 surface on the singular cohomology

$$
H^{*}(X, \mathbb{Q})=\bigoplus_{i=0}^{4} H^{i}(X, \mathbb{Q}) \cong \mathbb{Q}^{24}
$$


is a Mathieu representation with

$$
\mu(G)=\operatorname{dim} H^{*}(X, \mathbb{Q})^{G} \geq 5 .
$$

From this Mukai deduces that $G$ is isomorphic to a subgroup of $M_{23}$ with at least five orbits. In positive characteristic, if $G$ is wild, then the formula for the number of fixed points is no longer true and the representation of $G$ on the $l$-adic cohomology, $l \neq p$,

$$
H_{\mathrm{et}}^{*}\left(X, \mathbb{Q}_{l}\right)=\bigoplus_{i=0}^{4} H_{\mathrm{et}}^{i}\left(X, \mathbb{Q}_{l}\right) \cong \mathbb{Q}_{l}^{24},
$$

is not Mathieu in general. However, in our case we have the following.

Proposition 4.2. Let $G$ be a finite group acting symplectically on a $K 3$ surface $X$ over a field of characteristic 11 . Then the representation of $G$ on $H_{\mathrm{et}}^{*}\left(X, \mathbb{Q}_{l}\right), l \neq 11$, is a Mathieu representation with $\operatorname{dim} H_{\mathrm{et}}^{*}\left(X, \mathbb{Q}_{l}\right)^{G} \geq 3$.

Proof. Note that $\operatorname{rank} \operatorname{Pic}(X)^{G} \geq 1$, and the second assertion follows. It remains to prove that the representation is Mathieu. By Lemma 4.1 it is enough to show this for automorphisms of order 11. Let $g \in G$ be an element of order 11. We need to show that the character $\chi(g)$ of the representation on the $l$-adic cohomology $H_{\mathrm{et}}^{*}\left(X, \mathbb{Q}_{l}\right)$ is equal to $\epsilon(11)=2$. Since

$$
\chi(g)=\operatorname{Tr}\left(g^{*} \mid H_{\mathrm{et}}^{*}\left(X, \mathbb{Q}_{l}\right)\right),
$$

it suffices to show that $\operatorname{Tr}\left(g^{*} \mid H_{\mathrm{et}}^{2}\left(X, \mathbb{Q}_{l}\right)\right)=0$. In order to show this, we recall a result of Illusie $([6,3.7 .3])$ that the characteristic polynomial of $g^{*} \mid H_{\mathrm{et}}^{*}\left(X, \mathbb{Q}_{l}\right)$ has integer coefficients and is independent of $l \neq p=11$. Thus, $\operatorname{Tr}\left(g^{*} \mid H_{\mathrm{et}}^{2}\left(X, \mathbb{Q}_{l}\right)\right)=0$ if and only if $\operatorname{dim} H_{\mathrm{et}}^{2}\left(X, \mathbb{Q}_{l}\right)^{g}=2$. Now the result follows from Lemma 2.3 (ii).

\section{Determination of the groups}

In this section we determine all possible finite groups which may act symplectically and wildly on a $\mathrm{K} 3$ surface in characteristic 11 . We use only purely group-theoretic arguments.

Proposition 5.1. Let $G$ be a finite group having a Mathieu representation over $\mathbb{Q}$ or over $\mathbb{Q}_{l}$ for all prime $l \neq 11$. Then

$$
\# G=2^{a} \cdot 3^{b} \cdot 5^{c} \cdot 7^{d} \cdot 11^{e} \cdot 23^{f}
$$

for some $a \leq 7, b \leq 2, c \leq 1, d \leq 1, e \leq 1, f \leq 1$.

Proof. If the representation is over $\mathbb{Q}$, this is the theorem of Mukai [8, Theorem (3.22)]. In his proof, Mukai uses at several places the fact that the representation is over $\mathbb{Q}$. The only essential place where he uses this is Proposition (3.21), where $G$ is assumed to be a 2-group containing a maximal normal abelian subgroup $A$ and the case of $A=$ $(\mathbb{Z} / 4)^{2}$ with $\#(G / A) \geq 2^{4}$ is excluded by using the fact that a certain 2-dimensional representation of the quaternion group $Q_{8}$ cannot be defined over $\mathbb{Q}$. We use the fact that $G$ also admits a Mathieu representation on the 2-adic cohomology, and it is easy to see that the representation of $Q_{8}$ cannot be defined over $\mathbb{Q}_{2}$. 
The following lemma is of purely group-theoretical nature and its proof follows an argument employed by S. Mukai [8].

Lemma 5.2. Let $G$ be a finite group admitting a Mathieu representation over $\mathbb{Q}$ or over $\mathbb{Q}_{l}$ for all prime $l \neq 11$. Assume $\mu(G) \geq 3$. Assume that $G$ contains an element of order 11 , but no elements of order $>11$. Then the order of $G$ is equal to one of the following:

$$
11, \quad 5 \cdot 11, \quad 2^{2} \cdot 3 \cdot 5 \cdot 11, \quad 2^{4} \cdot 3^{2} \cdot 5 \cdot 11, \quad 2^{7} \cdot 3^{2} \cdot 5 \cdot 7 \cdot 11 .
$$

Proof. Since $G$ has no elements of order 23, by Proposition 5.1, we have

$$
\# G=2^{a} \cdot 3^{b} \cdot 5^{c} \cdot 7^{d} \cdot 11 \quad \text { for } a \leq 7, b \leq 2, c \leq 1, d \leq 1 .
$$

Let $S_{q}$ be a $q$-Sylow subgroup of $G$ for $q=5,7$ or 11 . Then $S_{q}$ is cyclic and its centralizer coincides with $S_{q}$. Let $N_{q}$ be the normalizer of $S_{q}$. Since $G$ does not contain elements of order $5 k, 7 k, 11 k$ with $k>1$, the index

$$
m_{q}:=\left[N_{q}: S_{q}\right]
$$

is a divisor of $\phi(q)=q-1$, where $\phi$ is the Euler function. Since it is known that the dihedral groups $D_{14}$ and $D_{22}$ do not admit a Mathieu representation, we have

$$
m_{7}=1 \text { or } 3 \text { and } m_{11}=1 \text { or } 5 .
$$

Let $a_{n}$ be the number of elements of order $n$ in $G$. Then

$$
a_{q}=\frac{\# G(q-1)}{q m_{q}} \quad \text { for } q=5,7,11 .
$$

As in [8], we have

$$
\mu(G)=\frac{1}{\# G} \sum \epsilon(n) a_{n}=8+\frac{1}{\# G}\left(16-2 a_{3}-4 a_{4}-4 a_{5}-6 a_{6}-5 a_{7}-6 a_{8}-6 a_{11}\right) .
$$

Case 1: The order of $G$ is divisible by 7 , i.e. $\# G=2^{a} \cdot 3^{b} \cdot 5^{c} \cdot 7 \cdot 11$. The formula (5.1) gives

$$
\mu(G) \leq 8+\frac{16}{\# G}-\frac{30}{7 m_{7}}-\frac{60}{11 m_{11}} .
$$

Since $\mu(G) \geq 3$, both $m_{7}$ and $m_{11}$ must be greater than 1 . Hence

$$
m_{7}=3, \quad m_{11}=5 .
$$

This implies that \# $G$ is divisible by 5 , and the formula 5.1 gives

$$
\mu(G) \leq 8+\frac{16}{\# G}-\frac{16}{5 m_{5}}-\frac{10}{7}-\frac{12}{11} .
$$

If $m_{5}=1$, then this inequality gives $\mu(G)<3$, a contradiction. 
If $m_{5}=2$, then the number of $q$-Sylow subgroups is equal to

$$
\frac{\# G}{q m_{q}}=2^{a-1} \cdot 3^{b} \cdot 7 \cdot 11(q=5), 2^{a} \cdot 3^{b-1} \cdot 5 \cdot 11(q=7), 2^{a} \cdot 3^{b} \cdot 7(q=11) .
$$

Taking $q=5$ and applying Sylow's theorem, we get

$$
2^{a-1} \cdot 3^{b} \cdot 7 \cdot 11 \equiv 1 \bmod 5, \quad \text { i.e. } a \equiv b \bmod 4 .
$$

Since $1 \leq a \leq 7$ and $1 \leq b \leq 2$, the only solutions are $(a, b)=(5,1),(6,2)$. However, neither $2^{5} \cdot 5 \cdot 11$ nor $2^{6} \cdot 3 \cdot \overline{5} \cdot 11$ is congruent to 1 modulo 7 .

If $m_{5}=4$, then the number of $q$-Sylow subgroups is equal to

$$
\frac{\# G}{q m_{q}}=2^{a-2} \cdot 3^{b} \cdot 7 \cdot 11(q=5), 2^{a} \cdot 3^{b-1} \cdot 5 \cdot 11(q=7), 2^{a} \cdot 3^{b} \cdot 7(q=11) .
$$

A similar argument shows that $a-b \equiv 1 \bmod 4$ and the possible order is

$$
\# G=2^{7} \cdot 3^{2} \cdot 5 \cdot 7 \cdot 11 .
$$

Case 2: The order of $G$ is divisible by 5 , but not by 7 , i.e. $\# G=2^{a} \cdot 3^{b} \cdot 5 \cdot 11$. The formula (5.1) gives

$$
\mu(G) \leq 8+\frac{16}{\# G}-\frac{16}{5 m_{5}}-\frac{60}{11 m_{11}} .
$$

If $m_{11}=1$, then this inequality gives $\mu(G)<3$, a contradiction. Hence

$$
m_{11}=5 \text {. }
$$

If $m_{5}=1$, then the number of $q$-Sylow subgroups is equal to $2^{a} \cdot 3^{b} \cdot 11(q=5)$, $2^{a} \cdot 3^{b}(q=11)$. By Sylow's theorem, $a-b \equiv 0 \bmod 4$ and $a+8 b \equiv 0 \bmod 10$. This system of congruences has only one solution $a=b=0$ in the range $a \leq 7, b \leq 2$. This gives the possible order

$$
\# G=5 \cdot 11 .
$$

If $m_{5}=2$, then the number of $q$-Sylow subgroups is equal to $2^{a-1} \cdot 3^{b} \cdot 11(q=5)$, $2^{a} \cdot 3^{b}(q=11)$. By Sylow's theorem, $a-b \equiv 1 \bmod 4$ and $a+8 b \equiv 0 \bmod 10$. Hence $a=2, b=1$, which gives the possible order

$$
\# G=2^{2} \cdot 3 \cdot 5 \cdot 11 .
$$

If $m_{5}=4$, then the number of $q$-Sylow subgroups is equal to $2^{a-2} \cdot 3^{b} \cdot 11(q=5)$, $2^{a} \cdot 3^{b}(q=11)$. By Sylow's theorem, $a-b \equiv 2 \bmod 4$ and $a+8 b \equiv 0 \bmod 10$. Hence $a=4, b=2$, giving the possible order

$$
\# G=2^{4} \cdot 3^{2} \cdot 5 \cdot 11 .
$$

Case 3: The order of $G$ is divisible by neither 5 nor 7 , i.e. $\# G=2^{a} \cdot 3^{b} \cdot 11$. In this case $m_{11} \neq 5$, and hence $m_{11}=1$. Thus the formula (5.1) gives

$$
\mu(G) \leq 8+\frac{16}{\# G}-\frac{60}{11} .
$$


The number of 11-Sylow subgroups is equal to $2^{a} \cdot 3^{b}$. By Sylow's theorem, $a+8 b \equiv$ 0 mod 10. This congruence has three solutions $(a, b)=(0,0),(2,1),(4,2)$ in the range $a \leq 7, b \leq 2$. In the second and the third case, the inequality (5.5) gives $\mu(G)<3$. The first gives the possible order

$$
\# G=11 .
$$

Proposition 5.3. In the situation of the previous lemma, $G$ is isomorphic to one of the following groups:

$$
C_{11}, \quad 11: 5, \quad L_{2}(11), \quad M_{11}, \quad M_{22} .
$$

Proof. By Lemma 5.2, there are five possible orders for $G$

$$
11, \quad 5 \cdot 11, \quad 2^{2} \cdot 3 \cdot 5 \cdot 11, \quad 2^{4} \cdot 3^{2} \cdot 5 \cdot 11, \quad 2^{7} \cdot 3^{2} \cdot 5 \cdot 7 \cdot 11 .
$$

In the first two cases, the assertion is obvious. The remaining possible three orders are exactly the orders of the three simple groups given in the assertion. The theory of finite simple groups shows that there is only one simple group of the given order in each of these cases.

Assume the last three cases. It suffices to show that $G$ is simple.

Let $K$ be a proper normal subgroup of $G$ such that $G / K$ is simple. If \#K is not divisible by 11 , then an order 11 element of $G$ acts on the set $\operatorname{Syl}_{q}(K)$ of $q$-Sylow subgroups of $K$. Since $\# \operatorname{Syl}_{q}(K)$ is not divisible by 11 for any prime $q$ dividing $\# K$, the order 11 element $g$ must normalize a $q$-Sylow subgroup of $K$. If one of the numbers $q=3,5$, or 7 divides $\# K$, then $g$ centralizes an element of one of these orders. This contradicts the assumption that $G$ does not contain an element of order $>11$. If $q=2$ divides \# $K$, then a 2-Sylow subgroup of $K$ is of order $\leq 2^{7}$, and hence $g$ centralizes an element of order 2, again a contradiction. So, we may assume that $11 \mid \# K$. If $\# K=11$, then an order 2 element of $G$ normalizes $K$. Neither a cyclic group of order 22 nor a dihedral group of order 22 has a Mathieu representation, so $\# K>11$. If $K \cong 11: 5$, then an order 2 element of $G$ normalizes the unique 11-Sylow subgroup of $K$, again a contradiction. If $\# K$ is one of the remaining three possibilities, then the group $G / K$ is of order $2^{5} \cdot 3 \cdot 7$ or $2^{3} \cdot 7$ or $2^{2} \cdot 3$. In the first case an order 7 element of $G$ normalizes, hence centralizes a Sylow 11-subgroup of $K$, again a contradiction. Obviously in the other two cases $G / K$ cannot be simple. This proves that $G$ is simple.

Corollary 5.4. Let $G$ be a finite group acting symplectically and wildly on a K3 surface $X$ over a field of characteristic 11. Let $g$ be an element of order 11 in $G$. Then the normalizer of $\langle g\rangle$ in $G$ must be isomorphic to $11: 5$ if $\# G>11$.

\section{Proof of the main theorem}

In this section we complete the proof of Theorem 1.1 announced in Introduction. It remains to prove assertion (ii). 
Lemma 6.1. Assume $\varepsilon \neq 0$. Let $G \subset \operatorname{Aut}\left(X_{\varepsilon}\right)$ be a finite wild symplectic subgroup. If an element $g \in G$ of order 11 leaves invariant the standard elliptic fibration with a $g$ invariant section, then $G=\langle g\rangle \cong C_{11}$ and $G$ is conjugate to $H_{\varepsilon}=\left\langle g_{\varepsilon}\right\rangle$. In particular, $H_{\varepsilon}$ is a maximal finite wild symplectic subgroup of $\operatorname{Aut}\left(X_{\varepsilon}\right)$.

Proof. Since $g$ leaves a section invariant, it must be a conjugate to $g_{\varepsilon}$. So up to conjugation, we may assume that $g$ leaves the zero section $S_{\varepsilon}$ invariant. Thus $g=g_{\varepsilon}$ by Proposition 3.8

Suppose $\langle g\rangle \neq G$. Let $N$ be the normalizer of $\langle g\rangle$ in $G$. Then $N \cong 11: 5$ by Corollary 5.4

We claim that $N$ leaves invariant the standard elliptic pencil $|F|$. It is enough to show that $h\left(F_{0}\right)=F_{0}$ for any $h \in N$, where $F_{0}=X^{g}$ is a cuspidal curve in $|F|$. In fact, for any $x \in F_{0}$, we have $h(x)=h g(x)=g^{i} h(x)$ for some $i$, so $h(x) \in X^{g}=F_{0}$, which proves the claim.

Next, we claim that $N$ leaves invariant the zero section $S_{\varepsilon}$. In fact, $h\left(S_{\varepsilon}\right)=h g\left(S_{\varepsilon}\right)=$ $g^{i} h\left(S_{\varepsilon}\right)$, so $g$ leaves invariant $h\left(S_{\varepsilon}\right)$, and hence $h\left(S_{\varepsilon}\right)=S_{\varepsilon}$ as $g$ cannot leave invariant two distinct sections by Lemma2.3(ii).

Now Proposition 3.8 gives a contradiction. Hence, $G=\langle g\rangle$.

Lemma 6.2. Let $G \subset \operatorname{Aut}\left(X_{0}\right)$ be a finite wild symplectic subgroup, isomorphic to $L_{2}(11)$. If an element $g \in G$ of order 11 leaves invariant both the standard elliptic fibration and a section, then $G$ is conjugate to $H_{0}$. In particular, if $G$ contains $g_{0}$ then $G=H_{0}$.

Proof. Replacing $G$ by a conjugate subgroup in $\operatorname{Aut}\left(X_{0}\right)$, we may assume that $g$ leaves invariant both the standard elliptic fibration and the zero section $S_{0}$, i.e. $g=g_{0}$. We need to prove that $G=H_{0}$.

Let $|F|$ be the standard elliptic fibration. Then $g\left(S_{0}\right)=S_{0}$ and $X^{g}=F_{0}$, a cuspidal curve in $|F|$.

Let $N$ be the normalizer of $\langle g\rangle$ in $G$. Then $N \cong 11: 5$. The same argument as in the proof of Lemma 6.1 shows that $N$ leaves invariant both the standard elliptic pencil $|F|$ and the zero section $S_{0}$. By Proposition $3.8, N \subset H_{0}$.

We have $N \subset G \cap H_{0}$. Suppose $G \cap H_{0}=N$. Consider the $G$-orbit of the divisor class $[F] \in \operatorname{Pic}\left(X_{0}\right)$,

$$
G([F])=\left\{h([F]) \in \operatorname{Pic}\left(X_{0}\right) \mid h \in G\right\} .
$$

Clearly $N$ acts on it. Note

$$
\# G([F])=[G: N]=12 .
$$

Thus $G([F])$ is the set of 12 different elliptic fibrations with a section. The automorphism $g$ cannot leave invariant an elliptic fibration other than $|F|$, hence fixes $[F]$ and has one orbit on the remaining 11 elliptic fibrations, which we denote by $\left[F_{1}\right], \ldots,\left[F_{11}\right]$.

Recall that $H_{0}$ leaves invariant the zero section $S_{0}$. The three divisor classes

$$
[F], \quad \sum_{j=1}^{11}\left[F_{j}\right], \quad\left[S_{0}\right]
$$


are $N$-invariant, and their intersection matrix is

$$
\left(\begin{array}{ccc}
0 & 11 m & 1 \\
11 m & 110 m & 11 b \\
1 & 11 b & -2
\end{array}\right)
$$

where $m=F \cdot F_{i}, b=S_{0} \cdot F_{i}, i \geq 1$. Its determinant is equal to

$$
242\left(m^{2}+b m\right)-110 m,
$$

which cannot be 0 for any positive integers $m$ and $b$. This implies that

$$
\mu(N)=2+\operatorname{rank} \operatorname{Pic}\left(X_{0}\right)^{N} \geq 5,
$$

a contradiction to the equality $\mu(N)=4$. This proves that $N$ is a proper subgroup of $G \cap H_{0}$. Since $N$ is a maximal subgroup of $G$, we have $G=H_{0}$.

Note that $\mu\left(M_{11}\right)=\mu\left(M_{22}\right)=3$ and $\mu\left(L_{2}(11)\right)=4$. Note also that $L_{2}(11)$ is isomorphic to a maximal subgroup of both $M_{11}$ and $M_{22}$.

The following proposition completes the proof of Theorem 1.1 (ii).

Proposition 6.3. Let $G \subset \operatorname{Aut}\left(X_{0}\right)$ be a finite wild symplectic subgroup. Assume that $G \cong M_{11}$ or $M_{22}$. Then no conjugate of $G$ in $\operatorname{Aut}\left(X_{0}\right)$ contains the automorphism $g_{0}$ given by (1.2). In other words, no element of $G$ of order 11 can leave invariant both the standard elliptic fibration and a section. In particular, $H_{0}$ is a maximal finite wild symplectic subgroup of $\operatorname{Aut}\left(X_{0}\right)$.

Proof. Suppose that a conjugate of $G$ contains $g_{0}$. Replacing $G$ by the conjugate, we may assume that $g_{0} \in G$.

Let $K$ be a subgroup of $G$ such that $g_{0} \in K \subset G$ and $K \cong L_{2}(11)$. Then by Lemma 6.2. $K=H_{0}$. Thus $g_{0} \in H_{0} \subset G$. Since $H_{0} \cong L_{2}(11)$ is a maximal subgroup of $G$, its normalizer subgroup $N_{G}\left(H_{0}\right)$ coincides with $H_{0}$.

Let $|F|$ be the standard elliptic fibration on $X_{0}$, and $S_{0}$ the zero section. Then $g\left(S_{0}\right)=$ $S_{0}$ and $X^{g}=F_{0}$, a cuspidal curve in $|F|$. Furthermore, both the section $S_{0}$ and the elliptic pencil $|F|$ are $H_{0}$-invariant (see Definition 3.7).

Consider the $G$-orbit of the divisor class $[F]$,

$$
G([F])=\left\{h([F]) \in \operatorname{Pic}\left(X_{0}\right) \mid h \in G\right\} .
$$

Consider the action of $H_{0}$ on it. By Proposition 3.8 the stabilizer subgroup $G_{[F]}$ of $[F]$ coincides with $H_{0}$. The automorphism $g_{0}$ cannot leave invariant two different elliptic fibrations, hence fixes $[F]$ and has orbits on the set $G([F]) \backslash\{[F]\}$ of cardinality divisible by 11 . This implies that $H_{0}$ fixes $[F]$ and has orbits on the set $G([F]) \backslash\{[F]\}$ of cardinality divisible by 11 . Write

$$
G([F])=\left\{[F]=\left[F_{0}\right],\left[F_{1}\right], \ldots,\left[F_{r-1}\right]\right\}
$$

where $r=\# G([F])=\left[G: H_{0}\right]$. Let $\mathcal{O}_{1} \cup \cdots \cup \mathcal{O}_{s}$ be the orbit decomposition of the index set $\{1, \ldots, r-1\}$ by the action of $H_{0}$. Since $H_{0}$ fixes $[F]$ and acts transitively 
on each $\mathcal{O}_{i}$, the intersection number $F \cdot F_{t}$ is constant on the orbit $\mathcal{O}_{i}$ containing $t$, i.e. $F \cdot F_{t}=m_{i}$ for all $t \in \mathcal{O}_{i}$. Note that the divisor

$$
\mathcal{F}=\sum_{j=0}^{r-1} F_{j}
$$

is $G$-invariant, and

$$
\mathcal{F}^{2}=\left(\sum_{j=0}^{r-1} F_{j}\right)^{2}=r F_{0} \cdot \sum_{j=0}^{r-1} F_{j}=r \sum_{i=1}^{s} m_{i} \# \mathcal{O}_{i}
$$

Next recall that $H_{0}$ leaves invariant the zero section $S_{0}$. Similarly we consider the $G$-orbit of the divisor class $\left[S_{0}\right]$,

$$
G\left(\left[S_{0}\right]\right)=\left\{h\left(\left[S_{0}\right]\right) \in \operatorname{Pic}\left(X_{0}\right) \mid h \in G\right\} .
$$

Let $G_{0}$ be the stabilizer subgroup of [ $S_{0}$. Since it contains $H_{0}$ and $H_{0}$ is maximal in $G$, we see that $G_{0}=H_{0}$ or $G_{0}=G$.

Assume $G_{0}=H_{0}$. Then all stabilizers are conjugate to $H_{0}$. Similarly to the above we claim that $g_{0} \in H_{0}$ fixes no elements of $G\left(\left[S_{0}\right]\right)$ other than $\left[S_{0}\right]$. If $g_{0} h\left(S_{0}\right)=h\left(S_{0}\right)$ for some $h \in G$, then $g_{0} \in h H_{0} h^{-1}$ and since all cyclic subgroups of order 11 in $H_{0}$ are conjugate inside $H_{0}$ we can write $\left(g_{0}\right)=h h^{\prime}\left(g_{0}\right) h^{\prime-1} h^{-1}$ for some $h^{\prime} \in H_{0}$. This implies $h h^{\prime} \in N_{G}\left(\left(g_{0}\right)\right)$. Since \# $N_{G}\left(\left(g_{0}\right)\right)=\# N_{H_{0}}\left(\left(g_{0}\right)\right)=55$ (see the proof of Lemma 5.2), we obtain $N_{G}\left(\left(g_{0}\right)\right)=N_{H_{0}}\left(\left(g_{0}\right)\right) \subset H_{0}$, hence $h \in H_{0}$ and $h\left(S_{0}\right)=S_{0}$. This proves the claim and shows that $H_{0}$ has orbits on the set $G\left(S_{0}\right) \backslash\left\{S_{0}\right\}$ of cardinality divisible by 11 . Write

$$
G\left(\left[S_{0}\right]\right)=\left\{\left[S_{0}\right],\left[S_{1}\right], \ldots,\left[S_{r-1}\right]\right\} .
$$

It is clear that the divisor

$$
\mathcal{S}=\sum_{j=0}^{r-1} S_{j}
$$

is $G$-invariant. Let $S_{0} \cdot F_{t}=b_{i}$ for $t \in \mathcal{O}_{i}$. Then we have

$$
\mathcal{F} \cdot \mathcal{S}=\left(\sum_{j=0}^{r-1} F_{j}\right) \cdot\left(\sum_{j=0}^{r-1} S_{j}\right)=r S_{0} \cdot \sum_{j=0}^{r-1} F_{j}=r\left(1+\sum_{i=1}^{s} b_{i} \# \mathcal{O}_{i}\right) .
$$

In either case $G \cong M_{11}$ or $M_{22}$, we know $\mu(G)=3$ and hence the two divisors $\mathcal{F}$ and $\mathcal{S}$ are linearly dependent in $\operatorname{Pic}\left(X_{0}\right)$. This implies

$$
\mathcal{F}^{2} \mathcal{S}^{2}=(\mathcal{F} \cdot \mathcal{S})^{2}
$$

Substituting from 6.1, 6.2), we get

$$
r\left(\sum_{i=1}^{s} m_{i} \# \mathcal{O}_{i}\right) \mathcal{S}^{2}=r^{2}\left(1+\sum_{i=1}^{s} b_{i} \# \mathcal{O}_{i}\right)^{2} .
$$


Since $\# \mathcal{O}_{i} \equiv 0 \bmod 11$ for all $i$ and $r \equiv 1 \bmod 11$, we have LHS $\equiv 0 \bmod 11$, but RHS $\equiv 1 \bmod 11$, a contradiction.

Assume $G_{0}=G$. Then the divisor $\mathcal{S}=S_{0}$ is $G$-invariant, and we have a simpler equality

$$
r\left(\sum_{i=1}^{s} m_{i} \# \mathcal{O}_{i}\right) \mathcal{S}^{2}=\left(1+\sum_{i=1}^{s} b_{i} \# \mathcal{O}_{i}\right)^{2}
$$

again a contradiction.

Remark 6.4. In [7] Kondō proves that the unique supersingular K3 surface $X$ with Artin invariant 1 admits symplectic automorphism groups $G \cong M_{11}$ or $G \cong M_{22}$. It follows from the previous results that any element $g \in G$ of order 11 leaves invariant an elliptic pencil without a $g$-invariant section. In fact, according to his construction of $G$ on $X$, one can show that $\operatorname{Pic}(X)^{g} \cong U(11)$, hence a $g$-invariant elliptic pencil has only an 11 section.

It is known that the Brauer group of a supersingular K3 surface is isomorphic to the additive group of the field $k$ [1]. It is well-known that the group of torsors of an elliptic fibration with a section is isomorphic to the Brauer group. We do not know which torsors admit a nontrivial automorphism of order $p$ (maybe all?). Nor do we know whether they define elliptic fibrations on the same surface $X_{0}$. Note that the latter could happen only for torsors of order divisible by $p=\operatorname{char}(k)$. It would be very interesting to see how the three groups $L_{2}(11), M_{11}$ and $M_{22}$ sit inside the infinite group $\operatorname{Aut}\left(X_{0}\right)$.

Remark 6.5. It follows from Lemma 3.5 that our surface $X_{0}$ admits a nonsymplectic automorphism of order 12. By Remark 3.6 $X_{0}$ is supersingular with Artin invariant $\sigma=1$. It follows from [9] that the maximal order of a nonsymplectic isomorphism of a supersingular surface with Artin invariant $\sigma$ divides $1+p^{\sigma}$. Thus 12 is the maximum possible order. What is the maximum possible nonsymplectic extension of $M_{11}$ or $M_{22}$ ?

Remark 6.6. A K 3 surface may admit a nonsymplectic automorphism of order 11 over any field of characteristic 0 or $p \neq 2,3,11$. The well-known example is the surface $V\left(x^{2}+y^{3}+z^{11}+w^{66}\right)$ in $\mathbb{P}(1,6,22,33)$. It would be interesting to know whether there exists a supersingular $\mathrm{K} 3$ surface $X$ which admits a nonsymplectic automorphism of order 11. It follows from [9] that, if $p \neq 2$, then 11 must divide $1+p^{\sigma}$, where $\sigma$ is the Artin invariant of $X$.

Acknowledgments. The authors are grateful to S. Kondō for many fruitful discussions. We also thank the referee for crucial comments, leading us to simplify the proof of Lemma 4.1.

Research of I. V. Dolgachev was partially supported by NSF grant DMS-0245203.

Research of J. Keum was supported by KRF grant KRF-2007-C00002.

\section{References}

[1] Artin, M.: Supersingular K3 surfaces. Ann. École Norm. Sup. 7, 543-567 (1974) Zbl 0322.14014 MR 0371899 
[2] Cossec, F., Dolgachev, I.: Enriques Surfaces I. Birkhäuser (1989) Zbl 0665.14017 MR 0986969

[3] Dolgachev, I., Keum, J.: Wild p-cyclic actions on K3-surfaces. J. Algebraic Geom. 10, 101131 (2001) Zbl 1044.14015 MR 1795552

[4] Dolgachev, I., Keum, J.: Finite groups of symplectic automorphisms of K3 surfaces in positive characteristic. Ann. of Math. 169, 269-313 (2009) Zbl pre05578745 MR 2480606

[5] Goto, Y.: The Artin invariant of supersingular weighted Delsarte surfaces. J. Math. Kyoto Univ. 36, 359-363 (1996) Zbl 0916.14019 MR 1411335

[6] Illusie, L.: Report on crystalline cohomology. In: Algebraic Geometry (Arcata, CA, 1974), Proc. Sympos. Pure Math. 29, Amer. Math. Soc., 459-478 (1975) Zbl 0326.14005 MR 0393034

[7] Kondō, S.: Maximal subgroups of the Mathieu group $M_{23}$ and symplectic automorphisms of supersingular K3 surfaces. Int. Math. Res. Notices 2006, art. ID 71517, 9 pp. Zbl 1099.14033 MR 2211150

[8] Mukai, S.: Finite groups of automorphisms of $K 3$ surfaces and the Mathieu group. Invent. Math. 94, 183-221 (1988) Zbl 0705.14045 MR 0958597

[9] Nygaard, N.: Higher de Rham-Witt complexes of supersingular K3 surfaces. Compos. Math. 42, 245-271 (1980/81) Zbl 0482.14009 MR 0596878

[10] Ogus, A.: Supersingular K3 crystals. In: Journées de Géométrie Algébrique de Rennes, Astérisque 64, 3-86 (1979) Zbl 0435.14003 MR 0563467

[11] Reid, M.: Chapters on algebraic surfaces. In: Complex Algebraic Geometry, IAS/Park City Math. Ser. 3, Amer. Math. Soc., 1-157 (1997) Zbl 0910.14016 MR 1442522

[12] Rudakov, A., Shafarevich, I.: Surfaces of type K3 over fields of finite characteristic. In: Current Problems in Mathematics 18, Akad. Nauk SSSR, Vsesoyuz. Inst. Nauchn. i Tekhn. Inform., Moscow, 115-207 (1981); reprinted in I. R. Shafarevich, Collected Mathematical Papers, Springer, 657-714 (1989) Zbl 0518.14015 MR 0633161

[13] Saint-Donat, B.: Projective models of K3 surfaces. Amer. J. Math. 96, 602-639 (1974) Zbl 0301.14011 MR 0364263

[14] Shioda, T.: Supersingular K3 surfaces. In: Algebraic Geometry (Copenhagen, 1978), Lecture Notes in Math. 732, Springer, Berlin, 564-591 (1979) Zbl 0414.14019 MR 0555718

[15] Shioda, T.: An explicit algorithm for computing the Picard number of certain algebraic surfaces. Amer. J. Math. 108, 415-432 (1986) Zbl 0602.14033 MR 0833362 